\title{
An Evaluation of the Key Indicator Based Surveillance System for International Health Regulations (IHR) -2005 Core Capacity Requirements in India
}

\author{
Vivek Singh*1, Jagan Mohan², U Prasada Rao², Lalit Dandona ${ }^{3,4}$ and David Heymann ${ }^{5}$
}

${ }^{1}$ Public Health Foundation of India (PHFI), Indian Institute of Public Health - Hyderabad, Hyderabad, India; ${ }^{2}$ Directorate of Health, Department of Health, Medical \& Family Welfare, Government of Andhra Pradesh, Hyderabad, India; ${ }^{3}$ Public Health Foundation of India, New Delhi, India; ${ }^{4}$ nstitute for Health Metrics and Evaluation, University of Washington, Seattle, WA, USA; ${ }^{5}$ London School of Hygiene and Tropical Medicine, London, United Kingdom

\section{Objective}

The goal of this study was to assess and review the factors (core and supplementary surveillance system attributes and functions) affecting the outcome of the Integrated Disease Surveillance Project (IDSP) in the state of Andhra Pradesh, in India.

\section{Introduction}

The IDSP is the key indicator based surveillance system in the country to fulfill the core surveillance and response functions requirement under the IHR-2005. Periodic reviews of the IDSP in the country have shown that it has been ineffective in meeting its main objective of disease control in the country. Efforts are required to systematically identify and adequately address the factors affecting the performance of IDSP for it to achieve its objectives and ensure compliance to the IHR-2005 requirements for national and global health security.

\section{Methods}

A cross-sectional survey was undertaken involving all 23 districts in the state. A composite score derived from the two key Surveillance Quality Indicators (SQIs) - Timeliness and Completeness of the weekly surveillance reports, was used to select 6 districts for visiting District Surveillance Units (DSUs) and 35 health facilities for conducting 110 Key Informant Interviews (KIIs), direct observations and secondary data analysis of surveillance records. The framework for the assessment was adapted from the frameworks proposed by the World Health Organization (WHO) and by Scott McNabb et al. for evaluation of surveillance systems.

\section{Results}

Overall, IDSP had infrastructure and mechanisms required for reporting but it functions mainly through reporting from health facilities under one of the health departments within the government health sector, the Directorate of Health, which governs and coordinates the project. There is inadequate participation of facilitates from other health departments and lack of inter departmental coordination at districts and state level. The involvement of private sector is very low. The laboratory capacity is week at all the levels of the system (Only 1 (17\%) out of 6 district laboratory is able to conduct all listed laboratory tests). Epidemiological data analysis (5\% KIIs) and outbreak investigation and response documentation is week. There are shortcomings in the process of training of personnel $(0 \%$ districts with all cadre strained) and supportive supervision activities (47\% KIIs had experienced any supervisory visit).

\section{Conclusions}

To meet the IHR-2005 core capacity requirements by 2015 the IDSP will need, 1. Greater coordination between all departments within the government health sector and increased involvement of the private health sector. 2 . Strengthening of laboratory capacity at all levels to achieve timely identification and response to outbreaks 3 . Regular human resource capacity building by training and supportive supervision, and 4. Adequate utilization of information, communication and technology to enhance epidemiological data analysis and supportive supervisory capability within the system.

\section{Keywords}

IHR-2005 Surveillance Core Capacity Requirement; Key Indicator Based Surveillance System India; Evaluation Findings

\section{Acknowledgments}

Authors acknowledge the participation of government health functionaries from the department of health, medical and family welfare, Government of Andhra Pradesh. This work was part of a fellowship grant through the Wellcome Trust Capacity Strengthening Strategic Award to the PHFI and consortium of UK Universities.

\section{References}

1. India - Integrated Disease Surveillance Project - Project Information Document. The World Bank, 2001 Contract No.: PID10512.

2. Suresh K. Integrated Diseases Surveillance Project (IDSP) through a consultant's lens. Indian journal of public health. 2008 JulSep;52(3):136-43. PubMed PMID: 19189835.

3. John TJ, Dandona L, Sharma VP, Kakkar M. Continuing challenge of infectious diseases in India. Lancet. 2011 Jan 15;377(9761):252-69. PubMed PMID: 21227500

4. Andrus JK, Aguilera X, Oliva O, Aldighieri S. Global health security and the International Health Regulations. BMC public health. 2010;10 Suppl 1:S2. PubMed PMID: 21143824. Pubmed Central PMCID: PMC3005574. Epub 2010/12/22. eng.

5. World Health Organization: Communicable disease surveillance and response system - guide to monitoring and evaluating World Health Organization, 2006.

6. McNabb SJ, Chungong S, Ryan M, Wuhib T, Nsubuga P, Alemu W, et al. Conceptual framework of public health surveillance and action and its application in health sector reform. BMC public health. 2002;2:2. PubMed PMID: 11846889. Pubmed Central PMCID: 65598.

*Vivek Singh

E-mail: vivek.singh@iiphh.org 